\title{
Portadores de bacterias multirresistentes de importancia clínica en áreas críticas (UCI-UCIN) de un hospital al norte del Perú
}

Aguilar Gamboa, Franklin Rómulo ${ }^{1}$, Aguilar Martinez, Sergio Luis² ${ }^{2}$ Cubas Alarcón, Deniss Manuel², Coaguila Cusicanqui, Luis Ángel ${ }^{3}$, Fernández Valverde, Darwin Almanzor ${ }^{1}$, Moreno Mantilla Mario Cecilio ${ }^{2}$, Román Campos Neftali ${ }^{3}$, Génesis Guevara Vásquez ${ }^{4}$, RobertoSegundo Díaz Sipión ${ }^{1}$

\section{RESUMEN}

Objetivo: Determinar la frecuencia de portadores de bacterias multirresistentes (BMR) y su perfil de resistencia en áreas críticas (UCI-UCIN) de un hospital público al norte del Perú (HRL).

Material y métodos: Se realizó un estudio observacional de corte transversal durante febrero - mayo 2015 en 48 pacientes y 32 personales de salud. Se consideraron 4 regiones anatómicas para el muestreo y emplearon medios suplementados con antibióticos para el aislamiento primario de BMR. El fenotipo de resistencia fue confirmado por los test de Jarlier, Hodge modificado y susceptibilidad a cefoxitin.

Resultados: La frecuencia de portadores de BMR en pacientes y personal asistencial fue del $79,1 \%$ y $3,1 \%$ respectivamente, además el $89,5 \%$ de pacientes ya eran portadores al momento de la admisión procedentes principalmente del servicio de emergencia, siendo la región ano-rectal la principal fuente de aislamientos con 75,0\%. Así mismo se determinó que la producción de betalactamasas de espectro extendido (BLEE) fue el principal fenotipo de resistencia con 77,65\%, seguido de la producción de carbapenemasas y meticilino resistencia con $14.2 \%$ y $8.24 \%$ respectivamente.

Conclusiones: Este estudio revela la alta frecuencia de portadores de bacterias multirresistentes en pacientes de áreas críticas del HRL y que la mayoría de estos ingresan ya colonizados principalmente en la región ano-rectal.

Palabras clave: farmacorresistencia bacteriana, portador sano, personal de salud.

\section{Carriers of multiresistant bacteria of clinical importance in critical areas (ICU - INCU) from a Hospital at north of Peru}

\section{ABSTRACT}

Objective: To determine the frequency of multiresistant bacteria Carriers (BM) and its profile of resistance in critical areas (ICU-INCU) in a public hospital in northern Peru (HRL).

Material and methods: A cross-sectional observational study was conducted during February to May 2015, in 48 patients and 32 healthcare staff. 4 anatomical regions for sampling were considered and supplemented instruments with antibiotics were used for primary isolation of BM. The resistance phenotype was confirmed by Jailer's test, Modified Hodge and susceptibility to cefoxitin.

Results: The frequencies of BM in patients and healthcare staff were $79.1 \%$ and $3.1 \%$ respectively; also $89.5 \%$ of patients were already carriers at the time of admission mainly from Emergency Service, being anorectal region the principal source of isolates with $75.0 \%$. It also was determined that production of extended spectrum beta-lactamase (ESBL) was the principal resistance phenotype with $77.65 \%$, followed by the production of carbapenemases and methicillin resistance with $14.2 \%$ and $8.24 \%$ respectively.

Conclusions: This study reveals the high frequency of multiresistant bacteria carriers in patients in critical areas of HRL and that most of these already were colonized in the anorectal region.

Key words: drug resistance, bacterial, carrier state, healthcare personnel.

1. Laboratorio de Microbiología, Dirección de Investigación, Hospital Regional Lambayeque, Lambayeque - Perú.

2. Laboratorio de Microbiología, Facultad de Ciencias Biológicas, Universidad Nacional Pedro Ruiz Gallo, Lambayeque - Perú.

3. Servicio de Unidades Críticas UCI-UCIN, Hospital Regional Lambayeque, Lambayeque - Perú.

4. Dirección de Investigación, Hospital Regional Lambayeque, Lambayeque - Perú. 
Aguilar Gamboa, Franklin Rómulo, Aguilar Martinez, Sergio Luis, Cubas Alarcón, Deniss Manuel, Coaguila Cusicanqui, Luis Ángel, Fernández Valverde, Darwin Almanzor, Moreno Mantilla Mario Cecilio, Román Campos Neftali, Génesis Guevara - Vásquez, Roberto Segundo Díaz Sipión

\section{INTRODUCCIÓN}

El estado portador es un evento común, resultante de la relación simbiótica comensal que establece un microorganismo con su hospedador sin que este le produzca signos o síntomas de infección, dicho microorganismo debe ser fácilmente transmisible a otro huésped susceptible ${ }^{(1)}$. Esta condición puede persistir durante días, meses o años, influenciado por la respuesta inmune, la competencia microbiana y el uso de antimicrobianos. Su importancia en el ámbito hospitalario radica en el hecho que existen microorganismos multirresistentes que pueden colonizar tanto al personal asistencial como a los pacientes; situación que se hace más evidente en aquellos que ingresan en áreas críticas, ya que son los más vulnerables de adquirir infecciones por su deprimida inmunidad y mecanismos invasivos a los cuales son sometidos como: sondas, catéteres, agujas y ventilación mecánica ${ }^{(2)}$.

La consecuencia más frecuente del estado portador es la infección intrahospitalaria. La cual es definida como toda infección que se adquiere y se manifiesta en un paciente después de 48-72 horas de haber ingresado a un centro hospitalario ${ }^{(3)}$. Esta es la mayor causa de muerte en países en vías de desarrollo, debida tanto a la severidad de la enfermedad subyacente que predispone a una mayor frecuencia de intervenciones invasivas así como al mayor uso de antibióticos de amplio espectro.

Las bacterias multirresistentes que pueden colonizar con mayor frecuencia a pacientes que ingresan en áreas críticas son Pseudomonas aeruginosa, Staphylococcus aureus, Escherichia coli, Klebsiella pneumoniae y el complejo Acinetobacter baumanniicalcoaceticus $^{(4,5)}$, por otro lado el personal de salud presenta en su mayoría una colonización por cocos gram positivos del género Staphylococcus ${ }^{(6)}$. Muchas veces se desconoce el mecanismo de transmisión de estos microorganismos y su fuente de diseminación dentro de este ámbito, cuestionándose el papel del ambiente hospitalario, el instrumental médico y equipos de ventilación como albergue de este tipo de bacterias, eludiendo muchas veces el papel fundamental que cumplen los portadores asintomáticos en la epidemiología de este tipo de infecciones.

El objetivo de este estudio fue estimar la frecuencia de portadores de bacterias multirresistentes y su perfil de resistencia en el personal de salud y pacientes que ingresaron a áreas críticas en un hospital al norte del Perú.

\section{MATERIAL Y MÉTODOS}

\section{Diseño y área de estudio}

Se realizó un estudio observacional de corte transversal entre febrero y mayo del 2015, en áreas críticas (UCI-UCIN) del hospital regional Lambayeque (HRL), Chiclayo- Perú. El HRL es un hospital de categoría III-1, que cuenta con áreas especializadas para la atención de pacientes que presentan patologías cuyo manejo requieren procesos de alta complejidad, esto en muchos casos implica el uso prolongado de antibióticos de amplio espectro lo cual genera la selección de bacterias multirresistentes de importancia clínica.

\section{Población y muestra}

La población estuvo constituida por el personal de salud y pacientes pertenecientes a UCI-UCIN del HRL. Se consideró como muestra a la totalidad de la población, la cual estuvo constituida por 80 personas, de las cuales 48 fueron pacientes y 32 personal de salud.

\section{Procedimiento}

La toma de muestra se realizó en paralelo para ambos grupos de estudio. En el caso del personal se tomaron 3 muestras en periodos diferentes considerando su disponibilidad y rol de servicios. Para los pacientes se consideró la toma de muestra en tres oportunidades: dentro de las $24 \mathrm{~h}, 72 \mathrm{~h}$ y a los 7 días de la admisión, previa firma del consentimiento informado por parte del familiar. Se consideraron 4 regiones anatómicas para el muestreo: nasal, faríngea, ano-rectal y palmar. En el caso del personal de salud no se consideró la ano-rectal mientras que para los pacientes no se consideró la palmar.

\section{Estudios microbiológicos}

Las muestras obtenidas fueron sembradas en medios de cultivo suplementados con antibióticos (agar manitol salado suplementado con oxacilina $5 \mathrm{mg} / \mathrm{l}$, agar Mc conkey y agar Cetrimide suplementado con cefotaxima $4 \mathrm{mg} / l)^{(7,8)}$ y fueron incubadas durante 18$24 \mathrm{~h}$ a $37^{\circ} \mathrm{C}$, luego se examinaron las características culturales y se procedió a la identificación microbiológica por métodos convencionales empleando TSI, LIA, Citrato de Simmons, MIO, y oxidasa para los bacilos Gram-negativos y catalasa y coagulasa para los cocos Gram-positivos. 
La susceptibilidad antimicrobiana fue evaluada mediante el método de difusión en disco siguiendo los parámetros de ensayo y rangos de interpretación propuestos por del manual CLSI $2014^{(9)}$. Así también se emplearon discos de cefoperazona/sulbactam (75/10ug) y debido a que éste no dispone de puntos de corte por parte del CLSI, se tomaron en cuenta los propuestos en otra investigación ${ }^{(10)}$. Cabe resaltar que los aislamientos con rango intermedio de susceptibilidad fueron considerados resistentes.

\section{Detección de fenotipos de resistencia bacteriana}

La confirmación de la producción de betalactamasas de espectro extendido tanto en Enterobacterias como en $P$. aeruginosa se realizó en paralelo con la determinación de corresistencias. Para Enterobacterias se utilizó el método de Jarlier, evaluando la presencia del sinergismo entre el disco de Amoxicilina/Ácido Clavulánico y los discos de ceftazidima, cefotaxima,ceftriaxona y aztreonam. Mientras que para $P$. aeruginosa se evaluó la presencia del sinergismo de los discos de Cefepime y Ceftazima frente a amoxicilina clavulanico ${ }^{(11)}$. Las placas se incubaron a $35^{\circ} \mathrm{C}$ durante $16-18 \mathrm{~h}$.

Para determinar la producción de carbapenemasas en el complejo Acinetobacter baumanniicalcoaceticus y Pseudomonas aeruginosa se realizó el Test de Hodge Modificado (MHT) ${ }^{(12)}$, usando una cepa de Klebsiella pneumoniae ATTC 700603 (cepa indicadora). Un resultado positivo se evidenció por el crecimiento de la cepa ATCC 700603 de Klebsiella pneumoniae en la parte de intersección entre el halo de inhibición generado por la difusión del antibiótico (meropenem) y la estría de la cepa problema formando una hendidura en la parte proximal al disco. Así mismo se realizó la detección fenotípica de cepas productoras de betalactamasas AmpC. Aunque no existen métodos fenotípicos estandarizados por el CLSI para la detección de enzimas AmpC, se realizó el método de aproximación de discos propuesto por Sanders y Sanders el cual posee una elevada sensibilidad y especificidad. La técnica consistió en realizar un antibiograma convencional, luego se colocó un disco de cefoxitin o cualquier otro antimicrobiano inductor a una distancia de $27 \mathrm{~mm}$ centro-centro de un disco de ceftazidima, ceftriaxona o cefotaxima. Si se observa un halo de inhibición truncado del antimicrobiano sustrato, testigo o revelador nos demuestra una prueba positiva de producción de betalactamasa inducible tipo $\mathrm{AmpC}^{(13)}$. En el caso de Staphylococcus aureus se realizó la detección de cepas meticilino-resistentes mediada por el gen mecA para lo cual se utilizó un disco de cefoxitin $30 \mathrm{ug}$, la placa fue incubada a $35^{\circ} \mathrm{C}$ por 16-18h. Una cepa mecA positiva se confirmó cuando el diámetro del halo de inhibición fue $\leq 21 \mathrm{~mm}$.

\section{Consideraciones éticas}

El estudio fue revisado y aprobado por el comité de ética de la dirección de investigación del HRL. La participación en el estudio del personal de salud fue voluntaria con la firma de un consentimiento informado, en el caso de los pacientes el consentimiento fue firmado por su familiar o apoderado.

\section{Análisis estadístico}

Para analizar los datos de la presente investigación se utilizó el software estadístico SPSS versión 20.0; el cual nos permitió codificar y transformar las variables cuantitativas a cualitativas así como corroborar la consistencia de los datos antes de trabajarlos. Por consiguiente para identificar la presencia de portadores, especie bacteriana multirresistente, tipo de muestra biológica, fenotipo de resistencia se utilizó la estadística descriptiva univariado (según el tipo de variables); y se construyeron tablas expresadas en porcentajes y gráficos de barras correspondientes. En cuanto a la frecuencia de datos entre dos variables (para responder nuestros objetivos); se realizó un análisis bivariado (tablas de contingencia) los cuales se expresaron en porcentajes. Además se realizó la prueba estadística de chi-cuadrado, un valor $\mathrm{p}<0,05$ fue considerado estadísticamente significativo.

\section{RESULTADOS}

En el grupo conformado por el personal de salud se determinó que sólo el $3,1 \%(n=1)$ era portador de alguna bacteria multirresistente, mientras que en los pacientes la frecuencia fue del $79,1 \%(n=38)$. Por otro lado el $89,5 \%(n=34)$ de pacientes ya era portador de bacterias multirresistentes al momento de la admisión, y solo un $10,5 \% \quad(n=4)$ lo adquirieron durante su estancia en áreas críticas (Tabla 1). 
Aguilar Gamboa, Franklin Rómulo, Aguilar Martinez, Sergio Luis, Cubas Alarcón, Deniss Manuel, Coaguila Cusicanqui, Luis Ángel, Fernández Valverde, Darwin Almanzor, Moreno Mantilla Mario Cecilio, Román Campos Neftali, Génesis Guevara - Vásquez, Roberto Segundo Díaz Sipión

Tabla 1. Características de los portadores identificados en el HRL - Hospital al norte del Perú

\begin{tabular}{|lcc|}
\hline Categorías & $\mathrm{n}$ & $\%$ \\
\hline Portadores & & \\
\hline Positivo & 38 & 79,1 \\
\hline Negativo & 10 & 20,9 \\
\hline Área & & \\
\hline Admisión & 34 & 89,5 \\
\hline Áreas críticas & 4 & 10,5 \\
\hline Muestra biológica & & \\
\hline Hisopado rectal & 36 & 75,0 \\
\hline Hisopado faríngeo & 30 & 62,5 \\
\hline Hisopado nasal & 22 & 45,8 \\
\hline Principales fenotipos & & \\
\hline BLEE & 66 & 77,7 \\
\hline CARBAPENEMASAS & 12 & 14,1 \\
\hline SARM & 7 & 8,2 \\
\hline
\end{tabular}

Fuente: Cuaderno de registro -Autores

E. coli $33.7 \%(n=28)$ y Klebsiella pneumoniae $27.7 \%$ $(\mathrm{n}=23)$ fueron las bacterias multirresistentes aisladas con mayor frecuencia en pacientes de áreas críticas; el área de $\mathrm{UCl}$ fue la que presentó el mayor número de aislamientos y la mayor frecuencia del estado portador se observó en el género masculino así mismo el rango de edad de 58-77 años presentó la mayor cantidad de aislamientos (Tabla 2).

Tabla 2. Bacterias aisladas según registros de los pacientes del HRL - Hospital al norte del Perú

\begin{tabular}{|c|c|c|c|c|c|c|}
\hline & $\begin{array}{c}\text { E.coli } \\
n=28\end{array}$ & $\begin{array}{c}K . \\
\text { pneumoni } \\
\text { ae } n=23\end{array}$ & $\begin{array}{c}A . \\
\text { bauman } \\
\text { nii } n=12\end{array}$ & $\begin{array}{c}P . \\
\text { aerugino } \\
\text { sa } n=11\end{array}$ & 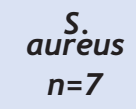 & $\begin{array}{c}\text { S. } \\
\text { maltophi } \\
\text { lia } n=2\end{array}$ \\
\hline Aislamientos $n=83$ & $28(33,7)$ & $23(27,7)$ & $12(14,5)$ & $11(13,3)$ & $7(8,4)$ & $2(2,4)$ \\
\hline \multicolumn{7}{|l|}{ Área } \\
\hline $\mathrm{UCl}$ & $20(52,6)$ & $16(42,1)$ & $7(18,4)$ & $7(18,4)$ & $5(13,2)$ & $2(5,3)$ \\
\hline UCIN & $8(21,1)$ & $7(18,4)$ & $5(13,4)$ & $4(10,5)$ & $2(5,3)$ & $0(0,0)$ \\
\hline \multicolumn{7}{|l|}{ Sexo } \\
\hline Femenino & $8(21,1)$ & $8(21,1)$ & $2(5,3)$ & $3(7,9)$ & $3(7,9)$ & $1(2,6)$ \\
\hline Masculino & $20(52,6)$ & $15(39,5)$ & $10(26,3)$ & $8(21,1)$ & $4(10,5)$ & $1(2,6)$ \\
\hline \multicolumn{7}{|l|}{ Edad } \\
\hline 18 a 37 años & $7(18,4)$ & $7(18,4)$ & $4(10,5)$ & $4(10,5)$ & $0(0,0)$ & $2(5,3)$ \\
\hline 38 a 57 años & $6(15,8)$ & $3(7,9)$ & $1(2,6)$ & $2(5,3)$ & $3(7,9)$ & $0(0,0)$ \\
\hline 58 a 77 años & $12(31,6)$ & $9(23,7)$ & $5(13,2)$ & $4(10,5)$ & $2(5,3)$ & $0(0,0)$ \\
\hline 78 a 97 años & $3(7,9)$ & $4(10,5)$ & $2(5,3)$ & $1(2,6)$ & $2(5,3)$ & $0(0,0)$ \\
\hline \multicolumn{7}{|l|}{ Mes } \\
\hline Marzo & $7(18,4)$ & $2(5,3)$ & $3(7,9)$ & $2(5,3)$ & $1(2,6)$ & $1(2,6)$ \\
\hline Abril & $15(39,5)$ & $15(39,5)$ & $5(13,2)$ & $4(10,5)$ & $3(7,9)$ & $1(2,6)$ \\
\hline Mayo & $6(15,8)$ & $6(15,8)$ & $4(10,5)$ & $5(13,2)$ & $3(7,9)$ & $0(0,0)$ \\
\hline
\end{tabular}

Fuente: Cuaderno de registro - Autores 
El único personal de salud portador presentó colonización por Klebsiella pneumoniae y pseudomonas aeruginosa en la región faríngea. En cuanto a los pacientes, se observó que la región anorectal representó la fuente de mayor número de aislamientos de bacterias multirresistentes con $75.0 \%(n=36)$, seguido de la región faríngea con un $62.5 \%(n=30)$ y de la nasal con $45.83 \%(n=22)$.

Los principales fenotipos de resistencia hallados se describen en la tabla I. Por otro lado E coli y K. pneumoniae presentaron elevada resistencia para ciprofloxacino y sulfametoxazol/ trimetoprim alcanzando frecuencias del $82.1 \%$ y $92.9 \%$ respectivamente. Los aminoglucósidos mostraron bajos niveles de resistencia sobretodo en $E$. coli alcanzando un $7.1 \%$. Así mismo ambas especies presentaron un $100 \%$ de sensibilidad a carbapenémicos (meropenem, imipenem) y cefoxitin.

El $90-100 \%$ del complejo Acinetobacter baumanniicalcoaceticus se caracterizó por elevados niveles de resistencia a quinolonas, aminoglucósidos, meropenem, sulfametoxazol/trimetoprim y tetraciclina, hallándose además un $63.6 \%$ y $81.8 \%$ de resistencia para tigeciclina y piperacilina/ tazobactam respectivamente. Mientras que en Pseudomonas aeruginosa, los niveles de resistencia a meropenem, cefepime, aztreonam y ciprofloxacino fueron del $81.8 \%$, encontrándose además una resistencia del $63.6 \%$ y $45.5 \%$ a amikacina y tetraciclina respectivamente, cabe resaltar que el $100 \%$ fue sensible a colistin e imipenem. Por último Staphylococcus aureus meticilino resistente presentó un $100 \%$ de resistencia a clindamicina, eritromicina y penicilina, hallándose además un $85.7 \%$ y $71.4 \%$ de resistencia a rifampicina y levofloxacino respectivamente.

\section{DISCUSIÓN}

El estado portador representa una importante fuente de diseminación de microorganismos, principalmente en el ámbito nosocomial. En este sentido la frecuencia de portadores observada en el presente estudio revela la preocupante circulación de BMR principalmente en los pacientes de áreas críticas del HRL, en los cuales se obtuvo un $79,1 \%$, cifra superior a la reportada en otros estudios ${ }^{(14)}$.

La frecuencia hallada puede estar relacionada con las condiciones propias de la hospitalización como la transmisión cruzada, estado inmunológico, comorbilidad, uso de dispositivos invasivos y la presión selectiva ejercida por los antibióticos, siendo estos últimos los más significativos considerando el uso extensivo de carbapenemes, glicopeptidos y polimixinas en estos servicios, así como el uso de dispositivos invasivos que producen cambios en la microbiota característica de cada paciente, debido a que proveen un ambiente ideal para la adherencia bacteriana y la formación de biofilm.

En cuanto a la frecuencia del estado portador en el personal de salud se halló sólo un 3,1\% de colonización por BMR. Esta cifra fue inferior a lo reportado en hospitales de Sudamérica, en los cuales se aisló principalmente SARM ${ }^{(15,16)}$. Cabe indicar que no fue motivo de la presente buscar asociación entre el estado portador en el personal de salud y en los pacientes de áreas críticas, así mismo las principales limitaciones del presente estudio fueron el período de muestreo y el número reducido de personal de salud participante, lo cual pudo influir en la baja frecuencia de portadores obtenida.

La detección de portadores a la admisión a un servicio revela el estado inicial de colonización de cada paciente e indica la adquisición de bacterias procedentes de otros servicios, motivo por el cual se suelen tomar muestras durante las primeras 24 horas de estancia en áreas críticas. En el presente se tomó este criterio y se pudo detectar un $89,5 \%$ de pacientes portadores de BMR a la admisión en áreas críticas del HRL procedentes principalmente del servicio de emergencia, lo que sugiere que el origen de estas bacterias podría no estar circunscrito a las unidades críticas sino que provienen de otros servicios hospitalarios.

La mayor cantidad de aislamientos de BMR, se obtuvieron de la región ano-rectal 75\%, y aunque en mucha literatura se encuentran tasas menores de colonización ${ }^{(17,18)}$, los resultados obtenidos se explicarían por el uso extendido de antiácidos en áreas críticas lo cual origina el cambio de $\mathrm{pH}$ del tracto gastrointestinal. Esto, sumado a la disminución del peristaltismo producto del estado prolongado de reposo y al uso de antibióticos de amplio espectro, crea las condiciones adecuadas para la colonización.

En cuanto a los portadores nasales y faríngeos, la frecuencia encontrada en la presente investigación fue del $45,83 \%$ y $62,5 \%$ respectivamente, dichos resultados son elevados en comparación con otros estudios similares ${ }^{(19,20)}$. Esta frecuencia puede estar 
Aguilar Gamboa, Franklin Rómulo, Aguilar Martinez, Sergio Luis, Cubas Alarcón, Deniss Manuel, Coaguila Cusicanqui, Luis Ángel, Fernández Valverde, Darwin Almanzor, Moreno Mantilla Mario Cecilio, Román Campos Neftali, Génesis Guevara - Vásquez, Roberto Segundo Díaz Sipión

relacionada con el uso de dispositivos invasivos, los cuales laceran la mucosa nasal y faríngea creando así un medio propicio para una posterior colonización bacteriana. Así mismo el uso de estos dispositivos interrumpe el reflejo de la tos, necesario para la expulsión de microorganismos patógenos del sistema respiratorio.

Es importante determinar las corresistencias que acompañan a los principales fenotipos de resistencia, pues se observa a nivel mundial un incremento presentándose como un fenómeno biológico que surge por el uso indebido y prolongado de fármacos; problema que se agudiza en los servicios de áreas críticas. En este sentido en el presente estudio se hallaron niveles de resistencia elevados para sulfametoxazol/trimetoprim y ciprofloxacino en aislamientos de Escherichia y Klebsiella pneumoniae productoras de BLEE con una marcada sensibilidad al meropenem $100 \%$. Este perfil de resistencia es muy frecuente en estos aislados microbianos en el Perú ${ }^{(21)}$, pudiéndose encontrar niveles más bajos de resistencia en otros países sudamericanos ${ }^{(22)}$.

El $90-100 \%$ de aislamientos del complejo Acinetobacter baumanni-calcoaceticus se caracterizaron por altas tasas de resistencia a quinolonas, ampicilina/sulbactam, aminoglucósidos, meropenem, sulfametoxazol/trimetoprim, tetraciclina y piperacilina/tazobactam, pero con un $100 \%$ de sensibilidad a Polimixina B. Este perfil de resistencia es reportado en diversas investigaciones ${ }^{(23)}$ y aunque este antibiótico se presente como una buena alternativa terapéutica frente a este tipo de microorganismos, el hecho que se presente como única elección y su uso extensivo condicionará la aparición de cepas resistentes en un futuro cercano, por lo cual deberían ensayarse nuevas alternativas terapéuticas.

En los aislamientos de Pseudomonas aeruginosa, se hallaron niveles elevados de resistencia a meropenem, cefepime, aztreonam, ciprofloxacino, cefoxitin, amikacina y tetraciclina, pero el $100 \%$ de dichos aislamientos fueron sensibles a colistin. Resultados que concuerdan con los perfiles de resistencia encontrados en otras investigaciones similares ${ }^{(23,24)}$. Mientras que los Staphylococcus aureus meticilino presentaron resistencia a clindamicina, eritromicina y penicilina en un $100 \%$, resultado que son similares a otros estudios ${ }^{(25)}$. Lo cual indica que los antibióticos antes mencionados no deben ser utilizados como terapia empírica en estos servicios.
Los gérmenes Gram negativos fueron los más aislados en las unidades estudiadas, siendo las enterobacterias $61,4 \%$ las de mayor representatividad dentro de este grupo, seguidos por bacilos no fermentadores $30,2 \%$ y SARM $8,4 \%$. Nuestros resultados coinciden con otros estudios donde encontraron altas tasas de aislamientos bacterianos correspondientes a bacilos Gram negativos; representados principalmente por E. coli y K. pneumoniae ${ }^{(22,26)}$. Aunque existen estudios que reportan mayores tasas de aislamientos de bacilos no fermentadores ${ }^{(27)}$. En este sentido cabe mencionar que los reportes de infecciones en enterobacterias comprende en general el $50 \%$ de los aislamientos hechos en pacientes con infecciones adquiridas en los hospitales y el $80 \%$ de todos los aislamientos de gérmenes gram negativos; destacando Klebsiella pneumoniae y Escherichia coli, como patógenos asociados especialmente en pacientes de unidades de cuidados intensivos ${ }^{(28)}$. Al respecto, es importante señalar que la tasa de colonización por estos microorganismos se incrementa hasta tres veces pues es influenciado por el ambiente hospitalario, la duración de la estancia y, especialmente, a la presión selectiva que ejercen los antibióticos sobre la flora comensal. Así mismo los bacilos gram negativos no fermentadores van cobrando notoria importancia por su incidencia en las infecciones hospitalarias destacando Acinetobacter baumannii y Pseudomonas aeruginosa quienes se comportan como patógenos oportunistas, pero que pueden causar severas infecciones especialmente en pacientes hospitalizados en áreas críticas.

En la presente investigación se logró determinar además que el estado portador en pacientes fue más frecuente en hombres con un $68,4 \%$, observándose además que el $42,1 \%$ de ellos presentaron un rango de edad de 58-77 años, la frecuencia de mayor colonización en el género masculino también ha sido reportada en otras investigaciones similares ${ }^{(29)}$; dejando abierto el debate del porque se suscita este evento.

Se logró determinar la producción de betalactamasas de espectro extendido (BLEE) en un 77,65\% distribuidas en los aislamientos de Escherichia coli, Pseudomonas aeruginosa y Klebsiella pneumoniae. Así mismo se detectó la presencia de carbapenemasas $14,12 \%$ en los aislamientos del complejo Acinetobacter baumannii-calcoaceticus y de meticilino resistencia $8,24 \%$ en Staphylococcus aureus (SARM). Resultados similares a los encontrados a nivel mundial en diversas investigaciones ${ }^{(17,30)}$ en 
Portadores de bacterias multirresistentes de importancia clínica

en áreas críticas (UCI-UCIN) de un hospital al norte del Perú

donde se determinó que el fenotipo BLEE resultó ser el principal mecanismo de resistencia; seguido del fenotipo AmpC derreprimido y carbapenemasa respectivamente. Además el $100 \%$ de SARM presentaron resistencia a cefoxitin lo cual corrobora la expresión del gen cromosómico denominado mecA. La detección de este gen es vital porque además contiene un elemento genético móvil denominado casete cromosómico estafilocócico que codifica la presencia de factores determinantes de resistencia adicionales a otros agentes antibacterianos, lo cual determina que los aislamientos SARM sean consideradas multirresistentes y presenten corresistencia a fluoroquinolonas, aminoglucósidos, macrólidos, azálidos, clindamicina y tetraciclinas.

En conclusión, este estudio revela la elevada frecuencia de portadores de bacterias multirresistentes en áreas críticas del HRL - Perú y que la principal fuente de las mismas se encuentra en la región ano-rectal de los pacientes, lo cual indica que esta es una importante fuente desde la cual se diseminarían este tipo de microorganismos. Así mismo, que un elevado porcentaje de pacientes llegan ya colonizados a los servicios de áreas críticas lo cual revela que estos microorganismos se estarían seleccionando en otros servicios o en la comunidad, y contradice la creencia comúnmente extendida de que el ambiente hospitalario y el personal de salud son las principales fuentes de los mismos, así mismo se halló que el principal fenotipo de resistencia fueron las BLEE, y en menor medida SARM y carbapenemasas, lo cual manifiesta la necesidad de realizar constantes estudios de vigilancia epidemiológica y estudio de portadores que nos permita anticipar la instauración de algún microorganismo en el servicio de áreas críticas de este hospital.

\section{REFERENCIAS BIBLIOGRÁFICAS}

1. Aguilar-Gamboa FR, Valiente JN, Mantilla MM. Portadores Nasofaríngeos de Staphylococcus aureus y Streptococcus pneumoniae en personal de Salud del Hospital Provincial Docente Belén de Lambayeque [Internet]. Revista Experiencia en Medicina del Hospital Regional Lambayeque. 2015 [cited 2016 Feb 6]. Available from: http://rem.hrlamb.gob.pe/ index.php/REM/article/view/17

2. López MJ, Cortés JA. Colonización e infección de la vía urinaria en el paciente críticamente enfermo. Med Intensiva [Internet]. Elsevier España, S.L.; [cited 2016 Feb 6];36(2):143-51. Available from: http://scielo.isciii.es/scielo.php?script= sci_arttext\&pid=S0210-56912012000200008\&lng =es\&nrm=iso\&tlng=es

3. Olaechea PM, Insausti J, Blanco A, Luque P. Epidemiología e impacto de las infecciones nosocomiales. Med Intensiva [Internet]. Elsevier España, S.L.; 2010 [cited 2014 Dec
3];34(4):256-67. Available from: http://scielo.isciii.es/ scielo.php?script $=$ sci_arttext\&pid $=$ S0210-5691201000 0400006\&lng=es\&nrm=iso\&tlng=es

4. Custovic A, Smajlovic J, Hadzic S, Ahmetagic S, Tihic N, Hadzagic $H$. Epidemiological surveillance of bacterial nosocomial infections in the surgical intensive care unit. Mater Sociomed [Internet]. 2014 Feb [cited 2014 Nov 26];26(1):7-11. Available from: http://www.pubmedcentral.nih.gov/ articlerender.fcgi?artid=3990379\&tool=pmcentrez\&rendertyp $\mathrm{e}=\mathrm{abstract}$

5. Geffers C, Gastmeier P. Nosocomial infections and multidrugresistant organisms in Germany: epidemiological data from KISS (the Hospital Infection Surveillance System). Dtsch Arztebl Int [Internet]. 2011 Feb [cited 2014 Nov 24];108(6):87-93. Available from: http://www. pubmedcentral. nih.gov/articlerender.fcgi?artid=3047718\&tool=pmcentrez\&re ndertype=abstract

6. Moura JP de, Pimenta FC, Hayashida M, Cruz ED de A, Canini SRM da S, Gir E. Colonization of nursing professionals by Staphylococcus aureus. Rev Lat Am Enfermagem. Escola de Enfermagem de Ribeirão Preto / Universidade de São Paulo; 2011 Apr;19(2):325-31.

7. Eliecer Cano M, Ángeles Domínguez M., Ezpeleta C, Padilla B, Ramírez de Arellano E, Martínez-Martínez L. Cultivos de vigilancia epidemiológica de bacterias resistentes a los antimicrobianos de interés nosocomial. Enferm Infecc Microbiol Clin. 2008;26(4):220-9.

8. Del Castillo MC, López-Cerezo L, Casal M, Pascual A. Evaluation of chromID ESBL medium for detecting carriers of extendedspectrum beta-lactamase-producing enterobacteriaceae. Enferm Infecc Microbiol Clin. Elsevier; 2011 Jan;29(6):471-2.

9. CLSI. Performance Standards for Antimicrobial Susceptibility Testing; Twenty-Second Informational Supplement. M100-S24. Clinical and Laboratory Standards Institute. wayne, PA; 2014.

10. Kumar D, Singh AK, Ali MR, Chander Y. Antimicrobial Susceptibility Profile of Extended Spectrum B-Lactamase (ESBL) Producing Escherichia coli from Various Clinical Samples. Infect Dis (Auckl). 2014 Jan;7:1-8.

11. Piersigilli AL, Enrico MC, Bongiovanni ME, Bilbao LE, Martínez G, Ledesma EM. Aislados clínicos de Pseudomonas aeruginosa productores de ß-lactamasa de espectro extendido en un centro privado de Córdoba. Rev Chil infectología. Sociedad Chilena de Infectología; 2009 Aug;26(4):331-5.

12. Pasteran F, Veliz O, Rapoport M, Guerriero L, Corso A. Sensitive and specific modified Hodge test for KPC and metallo-betalactamase detection in Pseudomonas aeruginosa by use of a novel indicator strain, Klebsiella pneumoniae ATCC 700603. J Clin Microbiol. 2011 Dec;49(12):4301-3.

13. Rojas DDVM. Betalactamasas tipo AmpC: generalidades y métodos para detección fenotípica. Rev la Soc Venez Microbiol. Sociedad Venezolana de Microbiología; 2009;29(2):78-83.

14. JV Schaal, Pasquier P, Delacour H, Salvadori A, Jarrassier A, Renner JR, et al. Prevalence of colonization by multiresistant bacteria on admission to the ICU in the French military hospital in Kabul, Afghanistan. Critical Care. BioMed Central; 2013. P78.

15. Yazgi H, Ertek M, Ozbek A, Kadanali A. [Nasal carriage of Staphylococcus aureus in hospital personnel and the normal population and antibiotic resistance of the isolates]. Mikrobiyol Bul. Jan;37(2-3):137-42.

16. Trindade JP de A, Goulart Rodrigues É, de Sousa TK, Prado Palos MA, Vieira dos Santos S de L. Trabajadores del área de salud de un hospital oncológico colonizados por microorganismos multidroga resistentes. Enfermería Glob. Universidad de Murcia; 13(33):227-39.

17. Sękowska A, Gospodarek E, Kusza K. The prevalence of infections and colonisation with Klebsiella pneumoniae strains isolated in ICU patients. Anaesthesiol Intensive Ther. Jan;46(4):280-3. 
Aguilar Gamboa, Franklin Rómulo, Aguilar Martinez, Sergio Luis, Cubas Alarcón, Deniss Manuel, Coaguila Cusicanqui, Luis Ángel, Fernández Valverde, Darwin Almanzor, Moreno Mantilla Mario Cecilio, Román Campos Neftali, Génesis Guevara - Vásquez, Roberto Segundo Díaz Sipión

18. Kim J, Lee JY, Kim S Il, Song W, Kim J-S, Jung S, et al. Rates of fecal transmission of extended-spectrum B-lactamaseproducing and carbapenem-resistant Enterobacteriaceae among patients in intensive care units in Korea. Ann Lab Med. 2014 Jan;34(1):20-5.

19. Bakır Saygan S, Yașar H, Eras Z, Cesur S, Irmak H, Dilmen U, et al. Methicillin-resistant Staphylococcus aureus and vancomycin-resistant Enterococcus carriage rates in a neonatal intensive care unit. Mikrobiyol Bul. 2010 Jul;44(3):529-31.

20. Silvestri L, Solidoro A, Milanese M, Van Saene HKF, Fontana F, Gregori D, et al. Topical oropharyngeal vancomycin to control methicillin-resistant Staphylococcus aureus lower airway infection in ventilated patients. Minerva Anestesiol. 2010 Mar;76(3):193-202.

21. Colquechagua Aliaga F, Sevilla Andrade C, Gonzales Escalante E. Enterobacterias productoras de Betalactamasas de espectro extendido en muestras fecales en el Instituto Nacional de Salud del Niño, Perú [Internet]. Revista Peruana de Medicina Experimental y Salud Pública. 2015 [cited 2015 Jun 28]. Available from: http://www.rpmesp.ins.gob.pe:8080/ index.php/rpmesp/article/view/5228

22. Martínez Buitrago E, Hernández C, Pallares C, Pacheco R, Hurtado K, Recalde M. Frecuencia de aislamientos microbiológicos y perfil de resistencia bacteriana en 13 clínicas y hospitales de alta complejidad en Santiago de Cali Colombia. Infectio. Elsevier; 2014 Mar;18(01):3-11.

23. Sales V, Oliveira E, Célia R, Gonçalves F, Melo C. Microbiological analysis of inanimate surfaces in an Intensive Care Unit and patient safety. Rev Enferm Ref. Escola Superior de Enfermagem de Coimbra - Unidade de Investigação em Ciências da Saúde Enfermagem; 2014 Dec;IV (3):45-53.

24. Medell Gago M, Hart Casares M, Mora Diaz I. Acinetobacter baumannii versus Pseudomonas aeruginosa. Comportamiento en pacientes críticos con ventilación mecánica. Rev Cubana Med. 1999, Editorial Ciencias Médicas; 51(3):239-46.

25. Paz Rojas EL, de León Pandolfi DP, Ramírez Ponce R. Resistencia bacteriana en cuidados intensivos y tendencia actual: Departamento de Cuidados Críticos, Servicio de Cuidados Intensivos del Hospital Nacional Guillermo Almenara Irigoyen, Essalud, Lima, Perú, 2004-2006. Acta Médica Peru. Colegio Médico del Perú; 25(3):140-7.

26. Hernández-Gómez C, Blanco VM, Motoa G, Correa A, Maya JJ, De la Cadena E, et al. Evolución de la resistencia antimicrobiana de bacilos Gram negativos en unidades de cuidados intensivos en Colombia. Biomédica [Internet]. 2013 Nov 5 [cited 2015 Jun 28];34:91. Available from: http://www.revistabiomedica.org/index.php/biomedica/arti cle/view/1667/2490
27. Trujillo Rodríguez Y, Fernández Alfonso JM, González Lorenzo A, López García I, Delgado Pérez L. Resistencia microbiana de gérmenes aislados en pacientes de las unidades de cuidados intensivos e intermedios. Hospital Universitario Clínico Quirúrgico Comandante Faustino Pérez. 2010. Rev Médica Electrónica. 1997, Centro Provincial de Información de Ciencias Médicas de Matanzas--FCMM; 34(5):509-20.

28. Benner KW, Prabhakaran P, Lowros AS. Epidemiology of infections due to extended-spectrum Beta-lactamaseproducing bacteria in a pediatric intensive care unit. J Pediatr Pharmacol Ther [Internet]. 2014 Apr [cited 2016 Feb 6];19(2):83-90. Available from: http://www.pubmedcentral. nih.gov/articlerender.fcgi?artid=4093669\&tool=pmcentrez\&r endertype=abstract

29. DalBen MF, Basso M, Garcia CP, Costa SF, Toscano CM, Jarvis WR, et al. Colonization pressure as a risk factor for colonization by multiresistant Acinetobacter spp and carbapenem-resistant Pseudomonas aeruginosa in an intensive care unit. Clinics (Sao Paulo) [Internet]. 2013 Jan [cited 2014 Nov 26];68(8):1128-33. Available from: http://www.pubmedcentral.nih.gov/ articlerender.fcgi?artid=3752633\&tool=pmcentrez\&rendertyp $\mathrm{e}=$ abstract

30. Nastro M, Montoto Piazza L, Saposnik E, García S, Barberis C, Vay $C$, et al. Resistencia a cefalosporinas de espectro extendido en enterobacterias sin AmpC inducible: Evaluación de los nuevos puntos de corte. Rev Argent Microbiol. Asociación Argentina de Microbiología; 44(1):30-5.

Fuentes de Financiamiento

Este artículo ha sido financiado por los autores y la Dirección de investigación del Hospital Regional Lambayeque-Perú.

\section{Conflictos de interés}

Los autores declaran no tener ningún conflicto de interés.

\section{Correspondencia:}

Franklin Rómulo Aguilar Gamboa

Dirección: Av. El progreso s/n.

Teléfono: 962074341

Correo electrónico: Krause_86@hotmail.com

Recibido: 27 de junio de 2016 Aprobado: 21 de julio de 2016 